\section{Laktoferrin, fäkales}

H. Fiedler

Erfurt, Deutschland

Englischer Begriff f(a)ecal lactoferrin

Definition Fäkales $\triangleright$ Laktoferrin ist ein Maß für die Chemotaxis-getriggerte Einwanderung von Granulozyten in die Mukosa und das Lumen des Darmes und besitzt keine Spezifität bezüglich der Grundkrankheit, aber erlaubt die Einschätzung der Stärke von Entzündungsvorgängen und deren Remissionen.

Beschreibung Fäkales Laktoferrin dient zum Nachweis und Ausschluss von akuten gastrointestinalen Entzündungen mit einer diagnostischen Sensitivität von ca. $90 \%$ und einer Spezifität von ca. $85 \%$. Wichtig für die Therapie ist die differenzielle Abgrenzung zu funktionellen Beschwerden, wie dem Reizdarmsyndrom. Das Monitoring entzündlicher Erkrankungen (M. Crohn, Colitis ulcerosa, Divertikulitis,
Mukoviszidose und Enteropathien durch nichtsteroidale Antirheumatika) korreliert mit klinischen, endoskopischen und histologischen Stadien. Gute Übereinstimmungen wurden bei Bestimmungen mit $>$ Calprotectin, weniger gut mit - PMN-Elastase gefunden. Die Diagnostik von Polypen und kolorektalen Tumoren ist umstritten (falsch positiv durch Blut im Stuhl).

Laktotransferrin ist im Stuhl einige Tage stabil, Postversand ist möglich. Es werden einzelne Stuhlproben oder Aliquote von Sammelstuhl verwendet. In Speziallabors werden die Proben aufbereitet und ELISAs eingesetzt. Als Entscheidungsgrenze für Entzündungen gilt $7 \mu \mathrm{g} / \mathrm{g}$ Stuhl. Säuglinge haben höhere Werte.

\section{Literatur}

Lehmann FS, Burri E, Beglinger C (2015) The role and utility of faecal markers in inflammatory bowel disease. Ther Adv Gastroenterol 8:23-36

Wright EK (2016) Calprotectin or lactoferrin: do they help. Dig Dis 34:98-104 